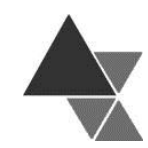

\title{
Alimentação saudável: prevenindo a síndrome metabólica
}

\author{
Maria Elisabeth Machado Pinto-e-Silva ${ }^{1}$, Paola Perino ${ }^{2}$, Bianca Guedes ${ }^{3}$, Thamires Leonardi \\ Merino Rios ${ }^{2}, V_{\text {Vanessa Sayuri Nagaishi }}{ }^{2}$ e Marlene Trigo ${ }^{4}$
}

A síndrome metabólica é um grupo de disfunções orgânicas que implica alterações nos níveis de glicemia, triglicérides, HDL-C e pressão arterial, levando ao desenvolvimento de doenças cardiovasculares, diabetes tipo 2 e obesidade. A alimentação saudável e as atividades físicas regulares ajudam a prevenir a síndrome metabólica. Este artigo tem como objetivo descrever uma ação educativa sobre a prevenção da síndrome metabólica por meio de recursos educacionais que estimulem o processo de conscientização dos indivíduos sobre a relevância de adotar um estilo de vida saudável. Com a participação de nutricionistas, esta ação educativa se concentrou em 57 funcionários não docentes de nível básico da universidade pública do município de São Paulo, entre dezembro de 2013 e março de 2014. Os tópicos abordados nos folhetos informativos foram "café da manhã", "como escolher o que comer", "o poder das frutas e legumes" e "proteger o seu coração". A avaliação, por meio de um questionário, mostrou que os folhetos contribuíram para a apreensão de informações, principalmente sobre a seleção de alimentos em refeições e a importância de tomar café da manhã.

Palavras-chave: Síndrome Metabólica; Alimentação Saudável; Educação Alimentar e Nutricional.

\section{Health eating: preventing the metabolic syndrome}

The metabolic syndrome is a group of organic dysfunctions which implies changes on the levels of glycaemia, triglycerides, HDL-C, and blood pressure, leading to the development of cardiovascular diseases, type 2 diabetes, and obesity. Health eating and regular physical activities help preventing the metabolic syndrome. This article aims at describing an educational action on metabolic syndrome prevention by educational resources that raise individuals' awareness on the relevance of adopting a healthier life style. With the help of nutritionists, this educational action focused on 57 non-teaching basic level employees of public university of city of San Pablo, between December 2013 and March 2014. The topics addressed were "breakfast", "how to choose what to eat", "the power of fruits and vegetables", and "protect your heart". The assessment through a questionnaire showed that the leaflets contributed to the knowledge about the issues, mainly on the selection of food and the importance of having breakfast.

Keywords: Metabolic Syndrome; Health Eating; Food and Nutrition Education.

${ }^{1}$ Livre Docente do Departamento de Nutrição, Faculdade de Saúde Pública, Universidade de São Paulo - USP, São Paulo, SP, Brasil. Endereço para correspondência: Departamento de Nutrição, Faculdade de Saúde Pública, Universidade de São Paulo - USP. Av. Dr. Arnaldo no 715, 2o andar, CEP: 01246-904, São Paulo, SP, Brasil. E-mail: mmachado@usp.br

2 Aprimoranda do Programa de Aprimoramento Profissional de Nutrição em Saúde Pública, Faculdade de Saúde Pública, Universidade de São Paulo - USP, São Paulo, SP, Brasil.

${ }^{3}$ Aluna de Graduação do Curso de Nutrição da Faculdade de Saúde Pública, Universidade de São Paulo - USP, São Paulo, SP, Brasil.

${ }^{4}$ Professora Sênior do Setor de Assistência para Assuntos Comunitários, Faculdade de Saúde Pública, Universidade de São Paulo - USP, São Paulo, SP, Brasil. 


\section{INTRODUÇÃO}

Inicialmente descrita por Reaven, em 1988, a síndrome metabólica é um conjunto de disfunções metabólicas, caracterizada pela resistência à insulina e hiperinsulinemia, com elevado teor de triglicérides e baixo nível de HDL e associada à hipertensão arterial sistêmica e obesidade ${ }^{[1]}$.

Embora o conceito de síndrome metabólica tenha obtido consenso em 2005 pela definição da International Diabetes Federation [2], a nomenclatura já havia sido definida pela Organização Mundial de Saúde, em $1998[3]$.

Assim, a adoção de hábitos alimentares saudáveis é essencial para a prevenção dessa síndrome. Entretanto, as pessoas se deparam com alguns questionamentos, como: “o que comer?”, "qual é o melhor alimento para cada refeição?", "qual a quantidade apropriada?", "o que não se deve consumir?". Um dos instrumentos para a busca das respostas é o Guia Alimentar para a População Brasileira, que possui diretrizes para promoção da alimentação saudável e apresenta metas para as famílias, profissionais de saúde, governos e setor produtivo de alimentos [4].

A elaboração de guias alimentares para populações é frequente desde a Primeira Guerra Mundial, tendo sido iniciada nos Estados Unidos. O material é elaborado a partir de inquéritos nutricionais, epidemiológicos e socioeconômicos. $\mathrm{Na}$ década de 1950, a maioria dos países da Europa e América possuíam seus guias alimentares no formato de círculos, chamados de rodas de alimentos, nos quais eram apresentados os grupos de alimentos [5,6]. A partir de 1992, os Estados Unidos passaram a adotar um modelo de pirâmide alimentar [7], incorporando-a em um novo modelo didático utilizado pelos guias alimentares. Esse modelo logo foi expandido para vários países, inclusive o Brasil, onde foi adaptado para nossa população ${ }^{[8]}$.

A promoção da alimentação saudável faz parte de um complexo de medidas preventivas, destacandose estratégias para chegar à população que carece de conhecimentos de nutrição. Os métodos para trabalhar a educação alimentar e nutricional com a população devem se preocupar em problematizar o assunto, a partir de princípios construtivistas e questões cotidianas, objetivando alcançar os membros da família como um todo [?].

O incentivo a hábitos de vida saudáveis que incluam atividades físicas e consumo de alimentos com baixa densidade energética e baixo teor de açúcar, gordura e sódio pode evitar que milhares de pessoas adoeçam [10]. Ou seja, o combate à síndrome metabólica requer um trabalho educativo que considere fatores psicológicos, socioeconômicos e ambientais, bem como a desinformação ou o excesso de informação sobre alimentação e nutrição, que dificultam o acesso a hábitos saudáveis [11,12].

A consciência sobre a necessidade de tomar atitudes para manter a boa saúde parece ser despertada apenas mediante o agravo à saúde [13]. A obesidade é epidêmica em várias partes do mundo, com mais de um bilhão de adultos com excesso de peso. Desses, 300 milhões já apresentam obesidade clínica [14]. Adicionalmente, o desenvolvimento da síndrome metabólica ocorre cada vez mais precocemente na população. Crianças já apresentam excesso de peso e níveis bioquímicos alterados, como elevação de glicemia, colesterol e triglicérides [15].

Para prevenir a obesidade, compete ao setor saúde identificar quais indivíduos estão ou estarão futuramente em risco nutricional devido ao sedentarismo e consumo insuficiente de frutas e verduras. A Organização Mundial de Saúde recomenda consumo diário de $400 \mathrm{~g}$ desses alimentos, sendo que quantidades inferiores aumentam o risco para doenças crônicas não-transmissíveis (DCNT) [16]. No Brasil, a mesma recomendação é adotada pelo Ministério da Saúde, via guias alimentares ${ }^{[4]}$.

O objetivo deste artigo é descrever uma ação educativa de prevenção da síndrome metabólica com potencial para estimular a reflexão dos indivíduos sobre seus estilos de vida e suas visões de vida saudável. 


\section{MATERIAL E MÉTODOS}

Foi realizado um projeto de extensão, destacando-se neste trabalho um estudo de intervenção nutricional com servidores de nível básico de uma universidade pública, do município de São Paulo. A coleta de dados foi conduzida entre maio de 2013 e abril de 2014. A caracterização da população foi realizada por meio de questionário sociodemográfico (renda, estado civil, escolaridade, ocupação) e avaliação nutricional (antropometria, recordatório de 24 horas e análises bioquímicas).

Peso, estatura e circunferência da cintura foram aferidos segundo as normas técnicas do Sistema de Vigilância Alimentar e Nutricional - SISVAN [17]. Os critérios para síndrome metabólica foram baseados no International Diabetes Federation [2], que estabelece circunferência da cintura elevada $(\geq 94 \mathrm{~cm}$ para homens $\mathrm{e} \geq 80 \mathrm{~cm}$ para as mulheres) como unidade fixa e elenca os demais indicadores da doença: triglicérides elevados $(\geq 150 \mathrm{mg} / \mathrm{dL})$, HDL reduzido $(\leq 40 \mathrm{mg} / \mathrm{dL}$ para homens e $\leq 50 \mathrm{mg} / \mathrm{dL}$ para mulheres), glicemia de jejum elevada ( $\geq 100 \mathrm{mg} / \mathrm{dL}$ ) e pressão arterial elevada $(\geq 130 / 85 \mathrm{mmHg})$. Quando três desses critérios estão presentes, caracteriza-se a síndrome metabólica. O consumo alimentar foi avaliado conforme os grupos de alimentos ingeridos, classificados em adequado e inadequado segundo as diretrizes do Guia Alimentar Brasileiro [4].

A intervenção foi realizada utilizando material gráfico em forma de folder educativo e entrega de ficha descritiva com os resultados da avaliação nutricional. O trabalho educativo foi realizado individualmente por nutricionista. Foram elaborados quatro folders educativos, com temas relevantes sobre nutrição e alimentação construídos a partir dos resultados obtidos por meio da avaliação nutricional. Esse material educativo teve por escopo ampliar o conhecimento dos servidores a respeito do próprio consumo alimentar e, desta maneira, melhorar as escolhas alimentares. Cada folder foi avaliado antes da entrega do seguinte, com um período de 15 a 20 dias entre um e outro. A avaliação constou de quatro questões de múltipla escolha sobre o conteúdo dos folders, sendo considerado satisfatório de três a quatro acertos (pelo menos 75\% de aproveitamento). Os valores médios relativos aos acertos manifestados pelos participantes (de acordo com o sexo) também foram calculados.

Esse estudo integra um projeto aprovado pelo Comitê de ética em Pesquisa da Instituição em 25/10/2013 (Processo no 516.520). Todos os participantes assinaram termo de consentimento livre e esclarecido.

\section{RESULTADOS}

\section{Caracterização dos servidores}

Dos 62 servidores de nível básico que trabalham na instituição, 57 aceitaram participar da intervenção. A média de idade foi de 51,6 anos. A maioria $(57,9 \%)$ era do sexo masculino e referiu ser casado ou ter companheira $(66,7 \%)$. A renda familiar média foi de $\mathrm{R} \$ 4.296,00$. Quanto à escolaridade, 14,0\% estudaram menos de 8 anos, $21,1 \%$ de 8 a 11 anos, $49,1 \%$ de 12 a 15 anos, e $15,8 \%$ com 16 ou mais anos. Os participantes se dividiam em três categorias profissionais: auxiliar administrativo $(54,4 \%)$, serviços gerais $(35,1 \%)$ e transporte $(10,5 \%)$.

\section{Avaliação nutricional}

Análise dos exames bioquímicos (Tabela 1) mostrou que glicemia de jejum elevada esteve presente em 24,6\% dos servidores. Triglicérides elevado foi mais prevalente em homens $(29,4 \%)$, assim como valores elevados de pressão arterial $(44,1 \%$, versus $21,7 \%$ de mulheres). HDL baixo foi observado em mais da metade dos homens $(55,9 \%)$ e em $65,2 \%$ das mulheres. Apresentaram circunferência da cintura elevada 76,5\% dos homens e em $87,0 \%$ das mulheres. Síndrome metabólica foi identificada em $38,6 \%$ da população, sendo maior em homens (41,2\%). 
Tabela 1. Descrição dos servidores segundo gênero e fatores de risco associados à Síndrome Metabólica e Índice de Massa Corporal elevado. São Paulo, SP, Brasil, 2014

\begin{tabular}{l|c|c|c|c|c|c}
\hline \multirow{2}{*}{ Fatores de Risco } & \multicolumn{2}{c|}{ Homem $(\mathrm{n}=34)$} & \multicolumn{2}{c|}{ Mulher $(\mathrm{n}=23)$} & \multicolumn{2}{c}{ Total (n=57) } \\
\cline { 2 - 7 } & $\mathrm{n}$ & $\%$ & $\mathrm{n}$ & $\%$ & $\mathrm{n}$ & $\%$ \\
\hline Glicemia de jejum elevada & 9 & 26,5 & 5 & 21,7 & 14 & 24,6 \\
TG elevado & 10 & 29,4 & 3 & 13,0 & 13 & 22,8 \\
HDL baixo & 19 & 55,9 & 15 & 65,2 & 34 & 59,6 \\
PA elevada & 15 & 44,1 & 5 & 21,7 & 20 & 35,1 \\
CC elevada & 26 & 76,5 & 20 & 87,0 & 46 & 80,7 \\
Síndrome metabólica & 14 & 41,2 & 8 & 34,8 & 22 & 38,6 \\
IMC: Sobrepeso & 19 & 55,9 & 12 & 52,2 & 31 & 54,4 \\
IMC: Obesidade & 8 & 23,5 & 6 & 26,1 & 14 & 24,6 \\
\hline TG: Trigcérides; HDI
\end{tabular}

TG: Triglicérides; HDL: high-density lipoprotein; PA: Pressão arterial; CC: Circunferência da cintura; IMC: Índice de Massa Corporal.

Ainda de acordo com a Tabela 1, o Índice de Massa Corporal - IMC revelou 79,0\% dos servidores com excesso de peso: $54,4 \%$ com sobrepeso e $24,6 \%$ com obesidade. Avaliação do consumo alimentar mostrou que $57,0 \%$ dos servidores não ingeriam café da manhã.

A Tabela 2 apresenta os resultados dos alimentos consumidos pelos servidores em relação aos grupos de alimentos [4]. Cereais/tubérculo/raízes e leite/derivados apresentaram valores de inadequação acima de 90,0\%. Em relação ao consumo de frutas e legumes/verduras, $87,0 \%$ das mulheres apresentaram inadequação, enquanto para os homens os valores foram de $82,3 \%$ (frutas) e 88,2\% (legumes/verduras), respectivamente. A ingestão de carnes/ovos mostrouse inadequada para mais de $70,0 \%$ de mulheres e homens avaliados, sendo que o consumo médio foi de quase duas vezes o recomendado [4].

Tabela 2. Distribuição dos servidores segundo gênero e consumo dos grupos alimentares (média de ingestão; adequação ou inadequação em relação à recomendação). São Paulo, SP, Brasil, 2014

\begin{tabular}{|c|c|c|c|c|c|c|c|c|c|c|c|}
\hline \multirow{3}{*}{ Grupos de Alimentos } & \multirow{3}{*}{$\begin{array}{l}\text { Recomendações* } \\
\text { (porção) }\end{array}$} & \multicolumn{5}{|c|}{ Homem $(\mathrm{n}=34)$} & \multicolumn{5}{|c|}{ Mulher $(n=23)$} \\
\hline & & \multirow{2}{*}{$\begin{array}{l}\text { Ingestão } \\
\text { (média) }\end{array}$} & \multicolumn{2}{|c|}{ Adequada } & \multicolumn{2}{|c|}{ Inadequada } & \multirow{2}{*}{$\begin{array}{c}\text { Ingestão } \\
\text { (média) }\end{array}$} & \multicolumn{2}{|c|}{ Adequada } & \multicolumn{2}{|c|}{ Inadequada } \\
\hline & & & $\mathrm{n}$ & $\%$ & $\mathrm{n}$ & $\%$ & & $\mathrm{n}$ & $\%$ & $\mathrm{n}$ & $\%$ \\
\hline Cereais/tubérculos/raízes & $5-6$ & 3,5 & 2 & 5,9 & 32 & 94,1 & 2,8 & 0 & 0,0 & 23 & 100,0 \\
\hline Feijões/oleaginosas & 1 & 1,6 & 17 & 50,0 & 17 & 50,0 & 1,0 & 6 & 26,1 & 17 & 73,9 \\
\hline Frutas & $3-5$ & 1,3 & 6 & 17,6 & 28 & 82,3 & 1,4 & 3 & 13,0 & 20 & 87,0 \\
\hline Legumes/verduras & $3-5$ & 1,1 & 4 & 11,8 & 30 & 88,2 & 1,3 & 3 & 13,0 & 20 & 87,0 \\
\hline Leites/derivados & 3 & 1,2 & 2 & 5,9 & 32 & 94,1 & 1,3 & 2 & 8,7 & 21 & 91,3 \\
\hline Carnes/ovos & 1 & 1,9 & 10 & 29,4 & 24 & 70,6 & 1,8 & 5 & 21,7 & 18 & 78,3 \\
\hline Óleos/gorduras & 1 & 1,2 & 28 & 82,3 & 6 & 17,6 & 1,1 & 21 & 91,3 & 2 & 8,7 \\
\hline Açúcares/doces & 1 & 0,6 & 29 & 85,3 & 5 & 14,7 & 1,0 & 17 & 73,9 & 6 & 26,1 \\
\hline
\end{tabular}

* Guia Alimentar para a População Brasileira ${ }^{[1]}$ (porções recomendadas) 


\section{Descrição do material educativo}

Os folders foram impressos em cores, em formato A4 dobrável em três partes. Foram aplicadas técnicas de redação e linguagem gráfica direcionadas a melhorar a apreensão da informação pelo público [18]. No campo do texto verbal, utilizaram-se expressões simples de uso comum, sentenças curtas e tom próximo ao coloquial. A informação foi organizada em tópicos e distribuída entre texto corrido, gráficos e quadros. $\mathrm{O}$ texto visual empregou diferença hierárquica de tipologia, paleta de cores definida e figuras ilustrativas. As imagens encontram-se livres de direitos autorais ou licenciadas sob o padrão Creative Commons, que prescinde do pagamento de taxas para uso.

O primeiro folder (Figura 1) abordou o tema "Café da manhã: um hábito saudável para seu dia-a-dia" e tratou da importância da realização do desjejum. A imagem de um café da manhã adequado destacou quais grupos alimentares devem ser consumidos nessa refeição, além de imagem da pirâmide alimentar ${ }^{[8]}$, que foi destacada com o mesmo propósito.

O segundo folder (Figura 2) abordou "Como escolher o que comer: os grupos de alimentos e suas porções diárias para uma alimentação equilibrada" e teve como propósito a composição de uma refeição balanceada. No destaque desse material educativo, cada grupo alimentar (cereais/tubérculos/raízes, verduras/legumes, frutas, leite/derivados, feijões/oleaginosas, carnes/ovos, óleo/gorduras, açúcares/doces) foi discutido de acordo com porções recomendadas segundo o Guia Alimentar para a População Brasileira [4]. O prato ideal, no gráfico representado no folder, exemplifica uma refeição de almoço ou jantar equilibrado, destacando o grupo de verduras e legumes.

Figura 1. Folder café da manhã: um hábito saudável para o seu dia-a-dia

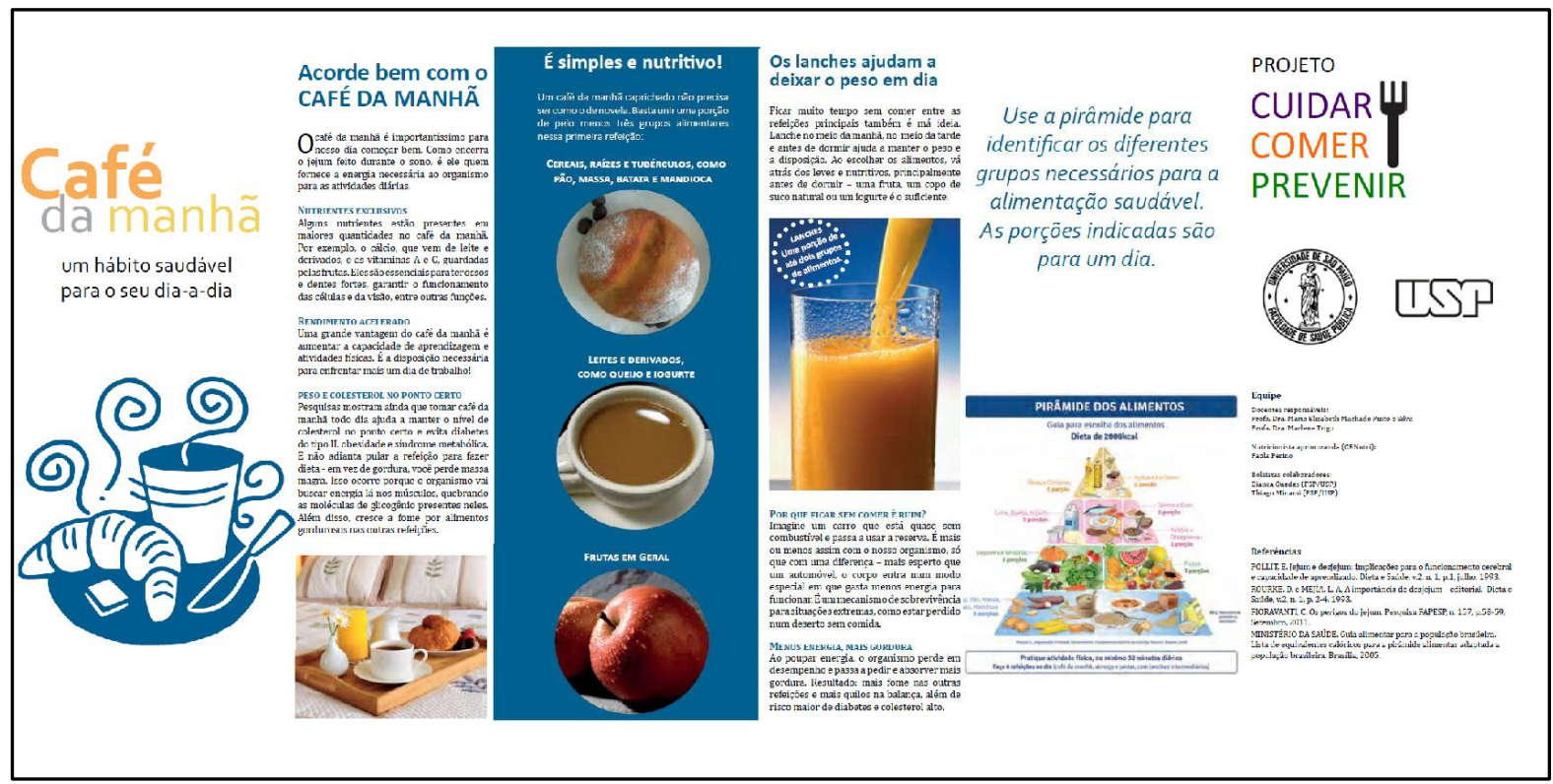


Figura 2. Folder Como escolher o que comer: os grupos de alimentos e suas porções diárias para uma alimentação diária

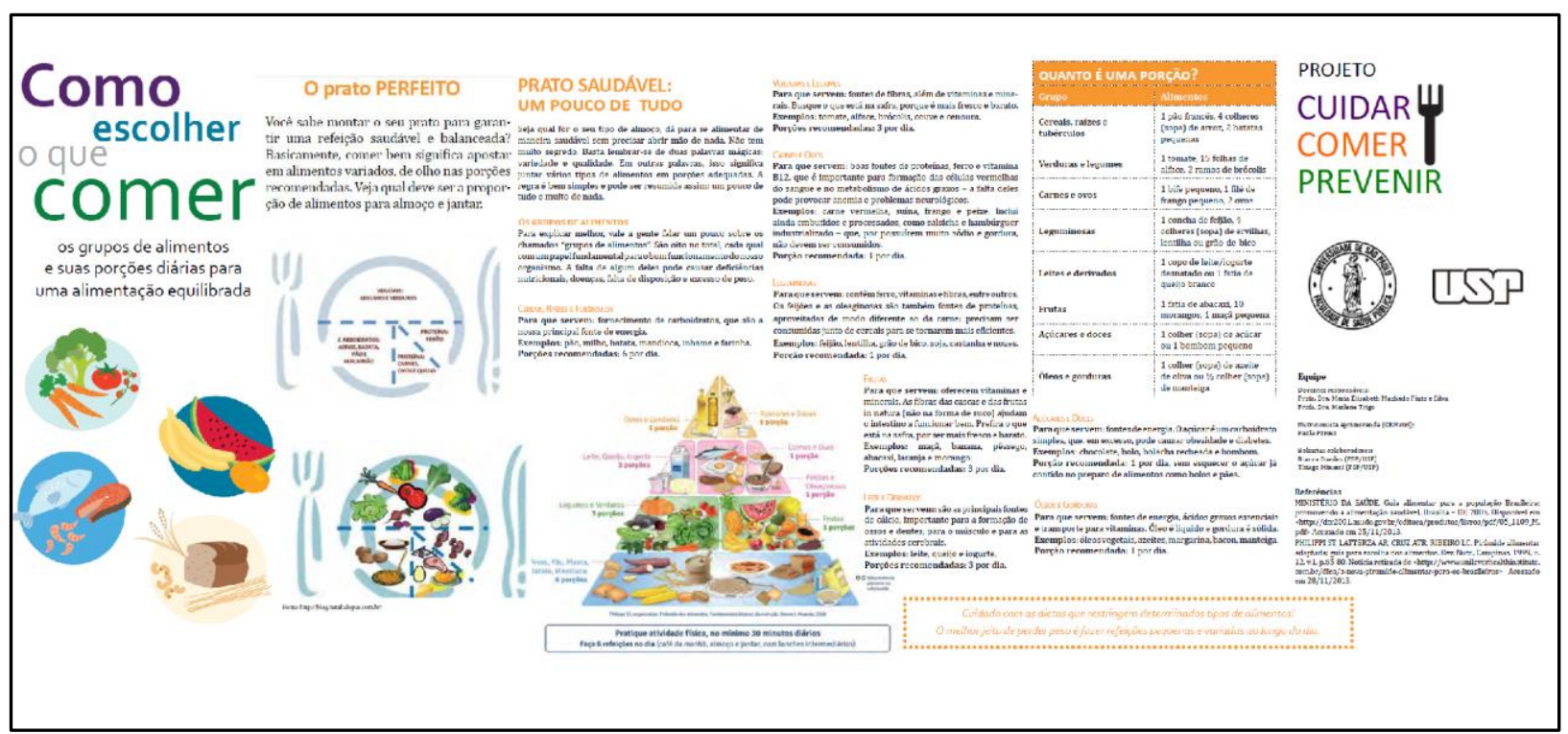

O terceiro folder (Figura 3), por sua vez, discutiu o tema "O poder das frutas e hortaliças: um manancial de vitaminas, fibras e minerais que ajudam a manter a saúde e o peso". Apresentou o consumo de frutas e verduras como medidas de prevenção de DCNT e abordou o teor de vitaminas e minerais nos alimentos. Também ofereceu informações sobre o consumo exagerado de carnes e gorduras na alimentação diária e suas consequências [19].

Figura 3. Folder $O$ poder das frutas e hortalicas: um manancial de vitaminas, fibras e minerais que ajudam a manter a saúde e peso

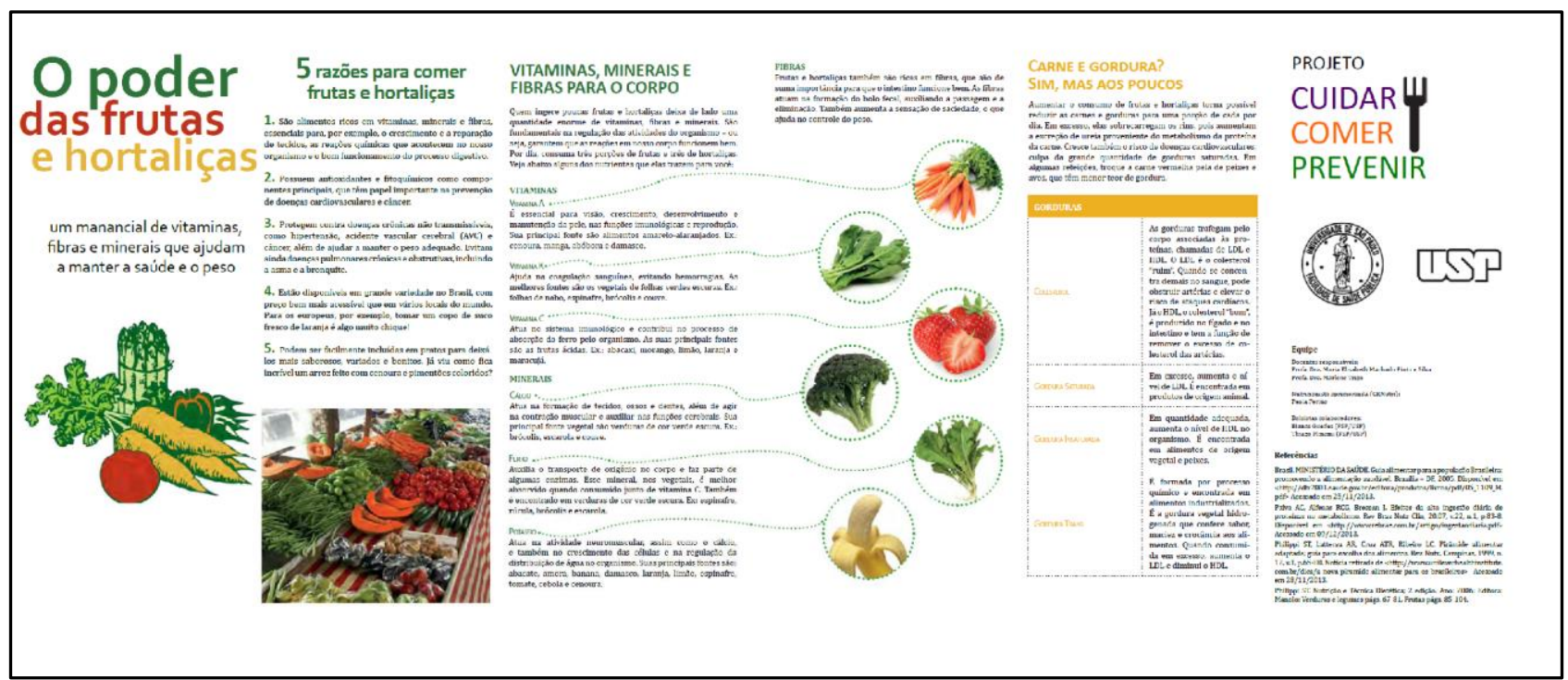


Por último, o quarto folder (Figura 4) elucidou o tópico "Proteja o coração: veja como evitar a síndrome metabólica, um transtorno que pode levar a doenças cardiovasculares e diabetes tipo 2". Tratou da prevenção da síndrome metabólica, apresentando os fatores causadores, diagnóstico e tratamento nãomedicamentoso. O folder apresenta a necessidade de ampliar o consumo diário de frutas, legumes e verduras e realizar atividade física por pelo menos 30 minutos diários.

Figura 4. Folder Proteja seu coração: veja como evitar a sindrome metabólica, um transtorno que pode levar a doenças cardiovasculares e diabetes tipo 2

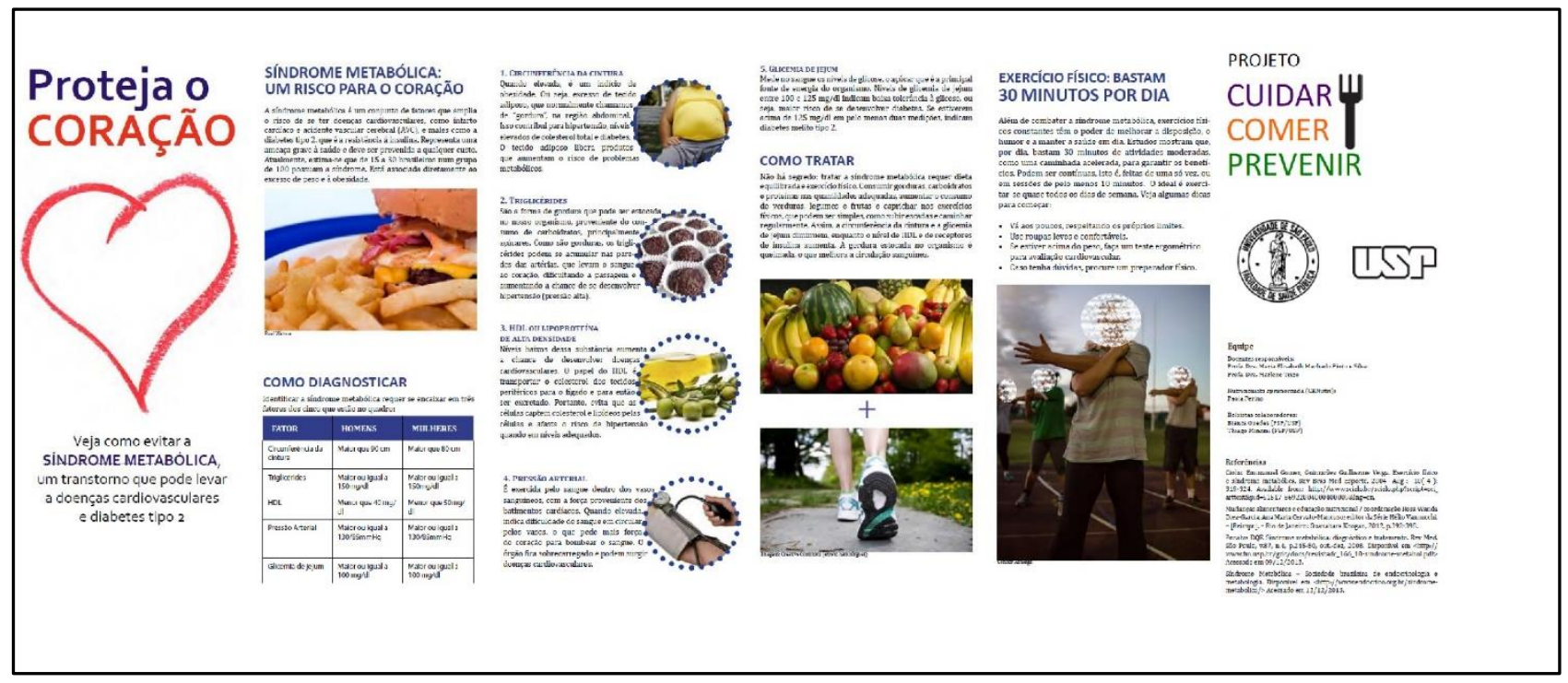

Os folders também foram disponibilizados aos participantes via endereço eletrônico e em meio online e gratuito. Todo o material está disponível para acesso público no seguinte endereço: http://cuidarcomerprevenir.tumblr.com

\section{Análise da compreensão dos folders}

O Gráfico 1 apresenta a análise da compreensão dos folders segundo anos de estudo.
Gráfico 1. Distribuição dos servidores segundo escolaridade e porcentagem de aproveitamento satisfatório ( 3 ou 4 acertos) para cada folder. São Paulo, SP, Brasil, 2014

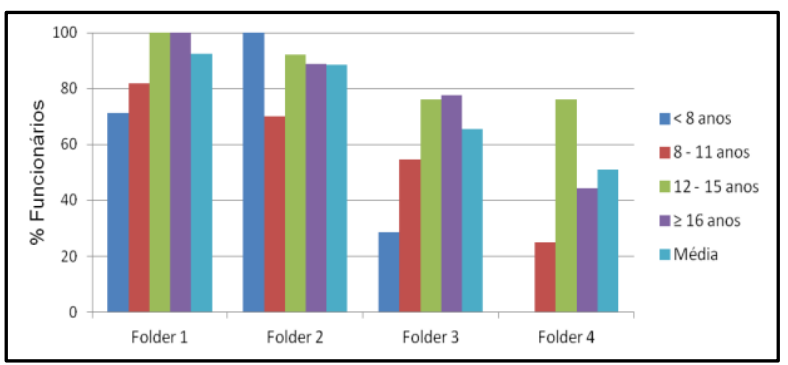


Considerando os quatro folders, foi observado que em média mais de 50\% obtiveram bom aproveitamento do material, independente do tempo de estudo (Gráfico 1). De modo geral, os dois grupos de maior escolaridade (12-15 anos e $\geq 16$ anos) apresentaram melhor desempenho na avaliação dos materiais, exceto para o folder 2 "Como escolher o que comer", que apresentou melhor aproveitamento pelos servidores com menos de 8 anos de estudo (100\% dos servidores acertaram 3 ou 4 questões).

Os servidores apresentaram maior dificuldade na avaliação do folder 4 "Proteja o coração", sendo observado que para todas as faixas de escolaridade foi obtido menor percentual de bom aproveitamento. Destaca-se que os indivíduos com nível superior completo ( $\geq 16$ anos, 44,4\% com aproveitamento satisfatório) apresentaram desempenho aquém daqueles com nível superior incompleto (12-15 anos, $76 \%$ ). Entre os funcionários com menos de 8 anos de estudo não houve bom aproveitamento, ou seja, nenhum pontuou 3 ou 4 acertos.

Tabela 3. Média de acertos na avaliação dos folders segundo gênero. São Paulo, SP, Brasil, 2014

\begin{tabular}{|c|c|c|c|c|c|c|}
\hline \multirow{2}{*}{ Gênero } & \multirow{2}{*}{$\mathrm{n}$} & \multicolumn{4}{|c|}{ Folder } & \multirow{2}{*}{ Média } \\
\hline & & 1 & 2 & 3 & 4 & \\
\hline Mulhere & 24 & 3,0 & 3,5 & 3,2 & 2,6 & 3,1 \\
\hline Homens & 33 & 3,0 & 3,2 & 2,8 & 2,4 & 2,9 \\
\hline
\end{tabular}

Quando analisado o desempenho dos servidores segundo gênero, são observados valores semelhantes referentes às médias de acertos (Tabela 3), sendo de 3,1 e 2,9 acertos para mulheres e homens, respectivamente.

\section{DISCUSSÃO}

O Guia Alimentar para a População Brasileira: Promovendo a Alimentação Saudável indica as recomendações de cada grupo alimentar, a fim de contribuir para uma nutrição adequada e, dessa forma, prevenir as DCNT [4]. Resumidamente, o Guia alimentar define como saudável a alimentação que contenha: a) frutas, legumes e verduras, que são ricos em vitaminas, minerais e fibras. São recomendadas três porções de frutas e três de legumes e verduras diariamente; b) uma porção de alimentos vegetais ricos em proteínas, particularmente as leguminosas, as sementes e as castanhas. Também é recomendada uma porção diária de carnes (de boi, porco, ave ou peixe, entre outras) e ovos, bem como três porções de leite e derivados, preferencialmente desnatados; c) alimentos com alta concentração de carboidratos, como os grãos (arroz, milho e trigo), pães, massas, tubérculos (batatas e inhame) e raízes (como a mandioca). Para garantir $45 \%$ a $65 \%$ da energia total diária da alimentação em forma de carboidratos, são necessárias seis porções diárias deste grupo; d) pouca quantidade de gorduras, açúcares e sal, sendo de $25 \%$ a $30 \%$ do total das calorias diárias advindas das gorduras e 10\% dos açúcares. Quanto ao sal, recomenda consumo diário máximo de 5g; e) o alimento essencial, a água, com consumo ideal de dois litros (seis a oito copos) por dia. Ainda, o Guia recomenda incentivar seu consumo entre as crianças e os idosos.

Analisando os grupos de alimentos que constituem o café da manhã (folder 1 ), o leite foi um dos alimentos com maior inadequação de consumo, bem como os cereais/tubérculos/raízes e as frutas. O café da manhã é considerado a refeição mais importante do dia. O consumo insuficiente de nutrientes (como proteínas, cálcio e vitaminas A e C) no café da manhã pode provocar alterações no organismo, uma vez que esses o protegem de agravos mais sérios, como a obesidade e suas consequências [20].

Não consumir o café da manhã pode aumentar a quantidade de alimento consumido pelo indivíduo na refeição seguinte. Com isso, há maior potencial de consumo excessivo e, consequentemente, acúmulo de gordura corporal. Aliado ao baixo gasto energético, isso dificulta a perda de peso corporal e favorece o surgimento de doenças cardiovasculares, diabetes, entre outras, pelo estabelecimento da síndrome metabólica [21,22].

Pesquisa Nacional do Ministério da Saúde sobre vigilância de fatores de risco e proteção para DCNT (Vigitel) mostrou que 48,1\% dos adultos apresentam excesso de peso $(52,1 \%$ dos homens e $44,3 \%$ das mulheres) [23]. Em São Paulo, o excesso de peso é de 51,1\% (55,9 em homens e 50,9\% em mulheres) [23]. Essas altas prevalências alertam ainda mais para a necessidade de adoção de alimentação saudável, visando a prevenção da obesidade e suas consequências. 
O objetivo do terceiro folder era estimular o aumento do consumo das frutas, legumes e verduras (FLV), destacando o poder das vitaminas e dos minerais. A Organização Mundial de Saúde recomenda consumo diário de $400 \mathrm{~g}$ desses alimentos, o que equivale a cinco porções [10]. Já o Guia alimentar indica três porções de frutas e três de legumes e verduras [4]. Neste estudo, os participantes apresentaram consumo insuficiente. De fato, isso ocorre com a maioria dos brasileiros [24,25]. No estudo do Vigitel [23], somente $36,6 \%$ dos moradores da cidade de São Paulo (29,7\% dos homens e $42,6 \%$ das mulheres) consumiam frutas e hortaliças cinco ou mais dias da semana. Ou seja, os alimentos com maior potencial de evitar a síndrome metabólica são justamente aqueles que mais faltam diariamente na alimentação da população.

O custo de FLV é, geralmente, fator limitante para seu consumo. Não basta o governo ter normas técnicas em manuais implantados em todas as unidades de saúde. O produtor desses alimentos deve estar envolvido pelo mesmo propósito de incentivar o consumo. Portanto, uma política que estimule o aumento da produção poderia auxiliar na solução desse

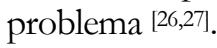

O grupo das gorduras e açúcares superou $80,0 \%$. As gorduras e os açucares estão presentes em grandes quantidades nos alimentos ultraprocessados [28], de fácil preparo e que podem ser estocados por muito tempo sem perder suas propriedades. $\mathrm{O}$ mesmo não ocorre com vegetais frescos, que precisam ser comprados duas a três vezes por semana e refrigerados a seguir $[29,30]$. Os guias alimentares para a população brasileira ${ }^{[4,28]}$ recomendam diminuição da adição de gorduras e açúcares nos alimentos, tanto pelo responsável pelo preparo dos alimentos nos domicílios, quanto pela indústria alimentícia. Pela pesquisa do Vigitel, 22,0\% (17,3\% dos homens e 26,0\% das mulheres) da população da cidade de São Paulo consomem doces em cinco ou mais dias da semana [23].

Quando as recomendações de cada grupo de alimentos são atendidas, a mortalidade por DCNT diminui. Assim, a educação em saúde torna-se fundamental para a redução da mortalidade na população [27,31,32,33]. Conhecer a pirâmide alimentar não é suficiente para que os indivíduos modifiquem o consumo e os hábitos alimentares. Os guias alimentares propostos são exemplos que devem ser trabalhados nos setores de saúde, educação, comércio e indústria.
Devem ser avaliados pelas áreas de marketing social e implantados nas instituições, na comunidade e no setor saúde (incluindo a atenção primária) para obter maior eficácia [34,35].

A avaliação do nível de apreensão das informações dos folders mostrou que os anos de escolaridade influenciaram o nível de compreensão do material educativo. $\mathrm{O}$ material mais complexo, o folder 4, atingiu maior percentual de acertos naqueles com mais anos de estudo. De fato, maior número de anos de estudo no ensino formal garante melhor rendimento intelectual e compreensão de textos e material gráfico ou digital [36].

A promoção da alimentação saudável exige um trabalho educativo. A maioria dos programas de educação nutricional não leva em conta fatores socioculturais e centra-se apenas em transmitir informações, estratégia considerada ineficaz. Este tipo de trabalho está fadado a não atingir os objetivos propostos nos projetos e não obter os resultados esperados, que é a transformação de maus hábitos alimentares em práticas saudáveis [12]. Quanto à forma de implantação do material educativo, o formato impresso é um método de baixo custo, utilizado frequentemente para consolidar a informação verbal passada por profissional de saúde e servir de referência posterior [36,37]. Observamos resultado satisfatório com o material proposto, que poderá servir como referência para o apoio à mudança de hábitos alimentares.

\section{CONCLUSÃO}

A ação educativa envolvendo a alimentação saudável e síndrome metabólica por meio de folders contribui positivamente para alertar as pessoas quanto à necessidade de adoção de hábitos saudáveis de vida. É o que mostra o alto índice de apreensão (curto prazo) das informações identificado neste estudo. O material gráfico, de forma geral, foi bem aceito pela maioria dos participantes. Avaliações em longo prazo são necessárias para mostrar mudanças decisivas na alimentação.

\section{AGRADECIMENTOS}

Ao Fundo de Cultura e Extensão Universitária (FCEx, da Universidade de São Paulo (USP) pelo financiamento do presente trabalho (no 432/2013). 
A Thiago Amaral Minami, aluno de pósgraduação em Nutrição em Saúde Pública da Faculdade de Saúde Pública - Universidade de São Paulo - USP pela confecção dos folders.

\section{REFERÊNCIAS}

[1] Reaven GM. Banting lecture 1988. Role of insulin resistance in human disease. Diabetes. 1988;37(12):1595-607. DOI http://dx.doi.org/10.2337/diab.37.12.1595

[2] International Diabetes Federation. IDF worldwide definition of the metabolic syndrome [internet]. Belgium (Brussel); 2006. [acesso em 30 set 2012]. Disponível em: http://www.idf.org/metabolic-syndrome

[3] Alberti KG, Zimmet PZ. WHO - World Health Organization. Definition, diagnosis and classification of diabetes mellitus and its complications. Part 1: diagnosis and classification of diabetes mellitus provisional report of a WHO consultation. Diabet. Med. 1998;15(7):539-553.

[4] Ministério da Saúde (BR). Secretaria de Atenção à Saúde. Coordenação Geral de Política de Alimentação e Nutrição. Guia alimentar para população brasileira: promovendo a alimentação saudável. Brasília (DF); 2005.

[5] Cervera P. Los grupos de alimentos en educación nutricional. Alimentaria. 1989; 26(201):25-29.

[6] Welsh S, Davis C, Shaw A. A brief history of food guides in the United States. Nutr. Today. 1992;27(6):6-11.

[7] US Department of Agriculture, Human Nutrition Information Service. Food guide pyramid. A guide to daily food choices. Washington (DC): Home Garden Bulletin. 1992;232:227.

[8] Philippi ST, Latterza AR, Cruz ATR, Ribeiro LC. Pirâmide alimentar adaptada: guia para escolha dos alimentos. Rev. Nutr. 1999;12:65-80.

[9] Teixeira PDS, Reis BZ, Vieira DAS, Costa D, Costa JO, Raposo OFF, et al. Intervenção nutricional educativa como ferramenta eficaz para mudança de hábitos alimentares e peso corporal entre praticantes de atividade física. Cien. Saúde. Colet. 2013;18(2):347-356. DOI http://dx.doi.org/10.1590/S141381232013000200006

[10] World Health Organization - WHO. The world health report 2002: Reducing risk, promoting healthy life. Geneva: WHO; 2002. (WHO Technical Report Series, 901).

[11] Vieira CM, Cordeiro SN, Magdaleno Júnior R, Turato ER. Significados da dieta e mudanças de hábitos para portadores de doenças metabólicas crônicas: uma revisão. Cien. Saúde. Colet. 2011;16(7):3161-3168. DOI http://dx.doi.org/10.1590/S141381232011000800016

[12] Waitzberg DL, Simopoulos AP, Bourne PG, Faergeman O. Obstáculos para implementação governamental de dietas saudáveis com base científica e como superá-los. Estud. Av. 2013;27(78):133-151. DOI http://dx.doi.org/10.1590/S010340142013000200010

[13] Castro IRR de, Castro LMC, Gugelmim AS. Ações educativas, programas e políticas envolvidos nas mudanças alimentares. In: Diez-Garcia RW, Cervato-Mancuso AM. Mudanças alimentares e educação nutricional. Rio de Janeiro: Guanabara-Koogan; 2012. p. 18-34.

[14] Organização Pan-Americana de Saúde. Doenças crônicas degenerativas e obesidade: estratégia mundial sobre alimentação saudável, atividade física e saúde. Brasília (DF); 2003.

[15] Pergher RNQ, Melo ME, Halpern A, Mancini MC. Liga de Obesidade Infantil. O diagnóstico de síndrome metabólica é aplicável às crianças? J. Pediatr. 2010;86(2):101-108. DOI http://dx.doi.org/10.2223/JPED.1983

[16] World Health Organization - WHO. Diet, nutrition and the prevention of chronic diseases. Report of a Joint WHO/FAO Expert Consultation. Geneva: WHO; 2003. (WHO Technical Report Series; 916).

[17] Ministério da Saúde (BR). Secretaria de Atenção à Saúde. Departamento de Atenção Básica. Orientação para coleta e análise dos dados antropométricos em Serviços de Saúde: Norma Técnica do Sistema de Vigilância Alimentar e Nutricional - SISVAN. Brasília (DF); 2011.

[18] Carrascoza JA. Razão e sensibilidade no texto publicitário. São Paulo: Futura; 2004.

[19] Ministério da Saúde (BR). Secretaria de Vigilância em Saúde. Departamento de Análise de Situação de Saúde. Plano de ações estratégicas para enfrentamento das doenças crônicas não transmissíveis (DCNT) no Brasil 2011-2022. Brasília (DF); 2011.

[20] Trancoso SC, Cavalli SB, Proença RPC. Café da manhã: caracterização, consumo e importância para a saúde. Rev. Nutr. 2010:23(5):859-869. DOI http://dx.doi.org/10.1590/S141552732010000500016

[21] Muff C, Reinhardt JD, Erbel R, Dragano N, Moebus S, Möhlenkamp S, et al. Who is at risk of irregular meal intake? Results from a population-based study. J. Public. Health. 2011;19(5):453-462. DOI http://dx.doi.org/10.1007/s10389011-0399-1 
[22] Timlin MT, Pereira MA. Breakfast frequency and quality in the etiology of adult obesity and chronic diseases. Nutr. Rev. 2007;65(6):268-281.

[23] Ministério da Saúde (BR). Secretaria de Atenção à Saúde. Departamento de Atenção Básica. Guia alimentar para a população brasileira. 2ed. Brasília (DF); 2014.

[24] Jaime PC, Figueiredo ICR, Moura EC, Malta DC. Fatores associados ao consumo de frutas e hortaliças no Brasil, 2006. Rev. de Saúde Pública. 2009;43(Supl 2):57-64. DOI http://dx.doi.org/10.1590/S0034-89102009000900008

[25] Mondini L, Moraes AS, Freitas ICM, Gimeno SGA. Consumo de frutas e hortaliças por adultos em Ribeirão Preto, SP. Rev. Saúde Pública. 2010;44(4):686-694. DOI http://dx.doi.org/10.1590/S0034-89102010000400012

[26] Claro R, Monteiro CA. Renda familiar, preço de alimentos e aquisição de frutas e hortaliças no Brasil. Rev. Saúde Pública. 2010;44(6):1014-1020. DOI http://dx.doi.org/10.1590/S003489102010000600005

[27] Lee JH, Ralston RA, Truby H. Influence of food cost on diet quality and risk factors for chronic disease: A systematic review. Nutr. Diet. 2011;68(4):248-261.

[28] Ministério da Saúde (BR). Secretaria de Vigilância em Saúde. Vigitel Brasil 2013: vigilância de fatores de risco e proteção para doenças crônicas por inquérito telefônico. Brasília (DF); 2014.

[29] Louzada MLC, Martins APB, Canella DS, Baraldi LG, Levy $\mathrm{RB}$, Claro RM, et al. Alimentos ultraprocessados e perfil nutricional da dieta no Brasil. Rev. Saúde Pública. 2015;49:38. DOI http://dx.doi.org/10.1590/S0034-8910.2015049006132

[30] Louzada MLC, Martins APB, Canella DS, Baraldi LG, Levy $\mathrm{RB}$, Claro RM, et al. Impacto de alimentos ultraprocessados sobre o teor de micronutrientes da dieta no Brasil. Rev. Saúde Pública. 2015;49(45). DOI http://dx.doi.org/10.1590/S00348910.2015049006211
[31] Castanho GKF, Marsola FC, Mclellan KCPM, Nicola M, Moreto F, Burini RC. Consumo de frutas, verduras e legumes associado à síndrome metabólica e seus componentes em amostra populacional adulta. Cien. Saúde Colet. 2013;18(2):385$392 . \quad$ DOI http://dx.doi.org/10.1590/S141381232013000200010

[32] Kothe EJ, Mullan BA. Perceptions of fruit and vegetable dietary guidelines among Australian young adults. Nutr. Diet. 2011;68(4):263-266. DOI http://dx.doi.org/10.1111/j.17470080.2011.01550.x

[33] Neutzling MB, Rombaldi AJ, Azevedo MR, Hallal PC. Fatores associados ao consumo de frutas, legumes e verduras em adultos de uma cidade no Sul do Brasil. Cad. Saúde Pública. 2009;25(11):2365-2374. DOI http://dx.doi.org/10.1590/S0102311X2009001100007

[34] Mathew EM, Rajiah K, Sharma K. Consumer's perception on design and layout of consumer medical information leaflets on obesity and lipid lowering drugs. J. Clin. Diagn. Res. 2013;7(12):2800-2802.

[35] Olivares Cortés S. Oportunidades y desafíos para la educación en nutrición utilizando las guías alimentarias en Chile. Perspect. Nut. Hum. 2009;11:107-117.

[36] McClinchy J, Dickinson A, Barron D, Thomas H. Practitioner and lay perspectives of the service provision of nutrition information leaflets in primary care. J. Hum. Nutr. Diet. 2011;24(6):552-559. DOI http://dx.doi.org/10.1111/j.1365277X.2011.01200.x

[37] Van der Zwaard BC, Poppe E, Vanwanseele B, Van der Horst HE, Elders PJ. Development and evaluation of a leaflet containing shoe advice: a randomized controlled trial. Fam. Pract. 2014;31(3):267-272. 